\title{
Comparison results for solutions to $p$-Laplace equations with Robin boundary conditions
}

\author{
Vincenzo Amato ${ }^{1} \cdot$ Andrea Gentile $^{2} \cdot$ Alba Lia Masiello $^{1}$ (D)
}

Received: 17 April 2021 / Accepted: 3 September 2021 / Published online: 15 September 2021

(c) The Author(s) 2021

\begin{abstract}
In the last decades, comparison results of Talenti type for Elliptic Problems with Dirichlet boundary conditions have been widely investigated. In this paper, we generalize the results obtained in Alvino et al. (Commun Pure Appl Math, to appear) to the case of $p$-Laplace operator with Robin boundary conditions. The point-wise comparison, obtained in Alvino et al. (to appear) only in the planar case, holds true in any dimension if $p$ is sufficiently small.
\end{abstract}

Keywords $p$-Lapacian $\cdot$ Robin boundary conditions

Mathematics Subject Classification $35 \mathrm{~J} 92 \cdot 35 \mathrm{P} 15$

\section{Introduction}

Let $\beta$ be a positive parameter and let $\Omega$ be a bounded open set of $\mathbb{R}^{n}, n \geq 2$, with Lipschitz boundary.

Let $f \in L^{p^{\prime}}(\Omega)$ be a non-negative function. We consider the following problem

$$
\begin{cases}-\operatorname{div}\left(|\nabla u|^{p-2} \nabla u\right)=f & \text { in } \Omega \\ |\nabla u|^{p-2} \frac{\partial u}{\partial v}+\beta|u|^{p-2} u=0 & \text { on } \partial \Omega .\end{cases}
$$

A function $u \in W^{1, p}(\Omega)$ is a weak solution to (1) if

Alba Lia Masiello

albalia.masiello@unina.it

Vincenzo Amato

vincenzo.amato@unina.it

Andrea Gentile

andrea.gentile2@unina.it

1 Dipartimento di Matematica e Applicazioni "R. Caccioppoli”, Universita ' degli Studi di Napoli

"Federico II", Complesso Universitario Monte S. Angelo, via Cintia, 80126 Naples, Italy

2 Mathematical and Physical Sciences for Advanced Materials and Technologies, Scuola Superiore Meridionale, Largo San Marcellino 10, 80126 Naples, Italy 


$$
\int_{\Omega}|\nabla u|^{p-2} \nabla u \nabla \varphi \mathrm{d} x+\beta \int_{\partial \Omega}|u|^{p-2} u \varphi \mathrm{d} \mathcal{H}^{n-1}(x)=\int_{\Omega} f \varphi \mathrm{d} x \quad \forall \varphi \in W^{1, p}(\Omega) .
$$

We want to establish a comparison principle with the solution to the following symmetrized problem

$$
\begin{cases}-\operatorname{div}\left(|\nabla v|^{p-2} \nabla v\right)=f^{\sharp} & \text { in } \Omega^{\sharp} \\ |\nabla v|^{p-2} \frac{\partial v}{\partial v}+\beta|v|^{p-2} v=0 & \text { on } \partial \Omega^{\sharp},\end{cases}
$$

where $\Omega^{\sharp}$ is the ball centered in the origin with the same measure of $\Omega$ and $f^{\sharp}$ is the Schwarz rearrangement of $f$ (see next section for its definition).

This kind of problems has been widely investigated in the last decades. The first step is contained in [10], where Talenti proved a pointwise comparison result between $u^{\sharp}$ and $v$ in the case of Dirichlet Laplacian. After this, several papers generalized the result of Talenti: for instance, the one by Talenti himself [11], in which the operator is a generic non-linear operator in divergence form, or the one by Alvino, Lions and Trombetti [2] in which the authors deal with both elliptic and parabolic cases: in both papers, Dirichlet boundary conditions are considered.

Different kinds of boundary conditions are considered by Alvino, Nitsch and Trombetti in [3], where they establish a comparison between a suitable norm of $u$ and $v$, respectively, solution to

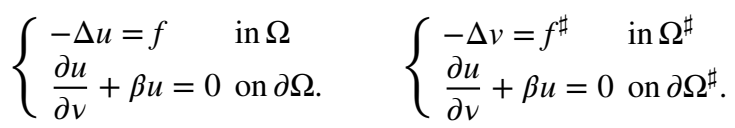

They found out that if $f$ is a non-negative function in $L^{2}(\Omega)$, then

$$
\begin{array}{cl}
\|u\|_{L^{k, 1}(\Omega)} \leq\|v\|_{L^{k, 1}\left(\Omega^{\sharp}\right)} & \forall 0<k \leq \frac{n}{2 n-2} \\
\|u\|_{L^{2 k, 2}(\Omega)} \leq\|v\|_{L^{2 k, 2}\left(\Omega^{\sharp}\right)} & \forall 0<k \leq \frac{n}{3 n-4}
\end{array}
$$

where $\|\cdot\|_{L^{k, q}}$ is the so-called Lorentz norm, whose definition can be found in next section. Moreover, the authors in [3] were able to establish a comparison á la Talenti,

$$
u^{\sharp}(x) \leq v(x), \quad \forall x \in \Omega^{\sharp}
$$

in the case $f \equiv 1$ and $n=2$. This will be the starting point of our work: it will be clear that our results coincide with the one in [3] in the case $p=2$.

For completeness sake, we cite that this wasn't the first result in this sense, indeed in [6] the authors study a comparison result for the $p$-torsion, that is the case $f \equiv 1$, with a completely different argument, obtaining

$$
\|u\|_{L^{1} \Omega} \leq\|v\|_{L^{1}\left(\Omega^{\sharp}\right)} .
$$

Another work that is worth to be mentioned is [1], where the authors obtained similar results to [3] in the case of mixed Dirichlet and Robin boundary conditions.

This paper is organized as follows. In the next section, we give some basic notions about rearrangements of functions and Lorentz spaces. Moreover, we list some properties of the solutions to problems (1) and (3). In Sect. 3, we prove the main results about comparison of the two solutions in terms of the Lorentz norm. 
In particular, we prove

Theorem 1.1 Let $u$ and $v$ be the solutions to problem (1) and (3), respectively. Then, we have

$$
\begin{gathered}
\|u\|_{L^{k, 1}(\Omega)} \leq\|v\|_{L^{k, 1}\left(\Omega^{\sharp}\right)} \forall 0<k \leq \frac{n(p-1)}{(n-1) p}, \\
\|u\|_{L^{p k, p}(\Omega)} \leq\|v\|_{L^{p k, p}\left(\Omega^{\sharp}\right)} \forall 0<k \leq \frac{n(p-1)}{(n-2) p+n} .
\end{gathered}
$$

We observe that from Theorem 1.1, we have that, if $p \geq n$

$$
\|u\|_{L^{1}(\Omega)} \leq\|v\|_{L^{1}\left(\Omega^{\sharp}\right)} \quad \text { and } \quad\|u\|_{L^{p}(\Omega)} \leq\|v\|_{L^{p}\left(\Omega^{\sharp}\right)} .
$$

Theorem 1.2 Assume that $f \equiv 1$ and let $u$ and $v$ be the solutions to (1) and (3), respectively.

(i) If $1 \leq p \leq \frac{n}{n-1}$ then

$$
u^{\sharp}(x) \leq v(x) \quad x \in \Omega^{\sharp},
$$

(ii) if $p>\frac{n}{n-1}$ and $0<k \leq \frac{n(p-1)}{n(p-1)-p}$, then

$$
\begin{aligned}
\|u\|_{L^{k, 1}(\Omega)} & \leq\|v\|_{L^{k, 1}\left(\Omega^{\sharp}\right)} \\
\|u\|_{L^{p, p}(\Omega)} & \leq\|v\|_{L^{p k, p}\left(\Omega^{\sharp}\right)} .
\end{aligned}
$$

Then, we explicitly observe that in the case $f \equiv 1$ from Theorem 1.2, we have that

$$
\|u\|_{L^{1}(\Omega)} \leq\|v\|_{L^{1}\left(\Omega^{\sharp}\right)} \quad \text { and } \quad\|u\|_{L^{p}(\Omega)} \leq\|v\|_{L^{p}\left(\Omega^{\sharp}\right)} \quad \text { for } p>1,
$$

while we have the point-wise comparison only for $p \leq \frac{n}{n-1}$.

In Sect. 4, using tools from Sect. 3, we give a new proof of the Faber-Krahn inequality with Robin boundary conditions in the case $p \geq n$. This topic was already studied in the papers by Bucur, Giacomini, Daners and Trebeschi, [4-6] and [7] where the authors proved the Faber-Krahn inequality for the eigenvalues of the Laplacian, or of the $p$-Laplacian, with Robin boundary conditions, for every $p>1$. Actually, the results in [4] are more general, since they hold for every $p>1$, but they are obtained with completely different tools than the ones contained in our paper.

Finally, in Sect. 5, we provide some examples and open problems, and we discuss the optimality of our results.

\section{Notions and preliminaries}

Definition 2.1 Let $u: \Omega \rightarrow \mathbb{R}$ be a measurable function, the distribution function of $u$ is the function $\mu:[0,+\infty[\rightarrow[0,+\infty[$ defined by 


$$
\mu(t)=|\{x \in \Omega:|u(x)|>t\}|
$$

Here, and in the whole paper, $|A|$ stands for the $n$-dimensional Lebesgue measure of the set A.

Definition 2.2 Let $u: \Omega \rightarrow \mathbb{R}$ be a measurable function, the decreasing rearrangement of $u$, denoted by $u^{*}$, is the distribution function of $\mu$.

The Schwarz rearrangement of $u$ is the function $u^{\sharp}$ whose level sets are balls with the same measure as the level sets of $u$. The functions $u^{\sharp}$ and $u^{*}$ are linked by the relation

$$
u^{\sharp}(x)=u^{*}\left(\omega_{n}|x|^{n}\right)
$$

It is easily checked that $u, u^{*} \mathrm{e} u^{\sharp}$ are equi-distributed, so it follows that

$$
\|u\|_{L^{p}(\Omega)}=\left\|u^{*}\right\|_{L^{p}(0,|\Omega|)}=\left\|u^{\sharp}\right\|_{L^{p}\left(\Omega^{\sharp}\right)} .
$$

An important propriety of the decreasing rearrangement is the Hardy-Littlewood inequaliy, that is

$$
\int_{\Omega}|h(x) g(x)| \mathrm{d} x \leq \int_{0}^{|\Omega|} h^{*}(s) g^{*}(s) \mathrm{d} s .
$$

So, by choosing $h=\chi_{\{|u|>t\}}$, one has

$$
\int_{|u|>t}|h(x)| \mathrm{d} x \leq \int_{0}^{\mu(t)} h^{*}(s) \mathrm{d} s .
$$

Definition 2.3 Let $0<p<+\infty$ and $0<q \leq+\infty$. The Lorentz space $L^{p, q}(\Omega)$ is the space of those functions such that the quantity:

$$
\|g\|_{L^{p, q}}= \begin{cases}p^{\frac{1}{q}}\left(\int_{0}^{\infty} t^{q} \mu(t)^{\frac{q}{p}} \frac{\mathrm{d} t}{t}\right)^{\frac{1}{q}} & 0<q<\infty \\ \sup _{t>0}\left(t^{p} \mu(t)\right) & q=\infty\end{cases}
$$

is finite.

Let us observe that for $p=q$, the Lorentz space coincides with the $L^{p}$ space, as a consequence of the well known Cavalieri's Principle

$$
\int_{\Omega}|g|^{p}=p \int_{0}^{+\infty} t^{p-1} \mu(t) \mathrm{d} t .
$$

See [12] for more details on Lorentz space.

Let us consider the functional

$$
\mathfrak{F}(w)=\frac{1}{p} \int_{\Omega}|\nabla w|^{p} \mathrm{~d} x+\frac{\beta}{p} \int_{\partial \Omega}|w|^{p} \mathrm{~d} \mathcal{H}^{n-1}(x)-\int_{\Omega} f w \mathrm{~d} x
$$


defined on $W^{1, p}(\Omega)$. This functional is well defined and its Euler-Lagrange equation is exactly (1). If we show that the functional admits a minimum, our problem will always have a solution.

(1) Let us show that the functional is bounded from below, indeed using the parametric Young inequality, we have

$$
\begin{aligned}
\mathfrak{F}(u) & \geq \frac{1}{p} \int_{\Omega}|\nabla u|^{p} \mathrm{~d} x+\frac{\beta}{p} \int_{\partial \Omega}|u|^{p} \mathrm{~d} \mathcal{H}^{n-1}(x)-\frac{\varepsilon^{p}}{p} \int_{\Omega}|u|^{p} \mathrm{~d} x-\frac{1}{p^{\prime} \varepsilon^{p^{\prime}}} \int_{\Omega}|f|^{p^{\prime}} \mathrm{d} x \\
& \geq \frac{1}{p}\left(\int_{\Omega}|\nabla u|^{p} \mathrm{~d} x+\beta \int_{\partial \Omega}|u|^{p} \mathrm{~d} \mathcal{H}^{n-1}(x)\right)-\frac{\varepsilon^{p}}{p} \int_{\Omega}|u|^{p} \mathrm{~d} x-\frac{1}{p^{\prime} \varepsilon^{p^{\prime}}} \int_{\Omega}|f|^{p^{\prime}} \mathrm{d} x \\
& \geq \frac{\lambda_{1, \beta}(\Omega)-\varepsilon^{p}}{p} \int_{\Omega}|u|^{p} \mathrm{~d} x-\frac{1}{p^{\prime} \varepsilon^{p^{\prime}}} \int_{\Omega}|f|^{p^{\prime}} \mathrm{d} x
\end{aligned}
$$

In the last inequality, we used the Sobolev inequality with trace term

$$
\int_{\Omega}|\nabla u|^{p}+\beta \int_{\partial \Omega}|u|^{p} \geq \lambda_{1, \beta}(\Omega) \int_{\Omega}|u|^{p} .
$$

In general, the quantity $\lambda_{1, \beta}(\Omega)$ denotes the first eigenvalue of the $p$-Laplacian with Robin boundary conditions, whose definition is given in (28), which can be also seen as a trace constant of the set $\Omega$.

If $\varepsilon$ is small enough, then the quantity

$$
\frac{\lambda_{1, \beta}(\Omega)-\varepsilon^{p}}{p}
$$

is non negative, and then

$$
\mathfrak{F}(u) \geq-\frac{1}{p^{\prime} \varepsilon^{p^{\prime}}} \int_{\Omega}|f|^{p^{\prime}}
$$

so

$$
m=\inf _{W^{1, p}} \mathfrak{F}(u)>-\infty .
$$

(2) Compactness and lower semicontinuity.

Let $\left\{u_{i}\right\}$ be a minimizing sequence. We can assume that $\mathfrak{F}\left(u_{i}\right) \leq m+1, \forall i$. Using again the Young inequality, we have

$$
\begin{aligned}
m+1 & \geq \frac{1}{p} \int_{\Omega}\left|\nabla u_{i}\right|^{p} \mathrm{~d} x+\frac{\beta}{p} \int_{\partial \Omega}\left|u_{i}\right|^{p} \mathrm{~d} \mathcal{H}^{n-1}(x)-\int_{\Omega} f u_{i} \mathrm{~d} x \\
& \geq \frac{1}{p} \int_{\Omega}\left|\nabla u_{i}\right|^{p} \mathrm{~d} x+\frac{\beta}{p} \int_{\partial \Omega}\left|u_{i}\right|^{p} \mathrm{~d} \mathcal{H}^{n-1}(x)-\frac{\varepsilon^{p}}{p} \int_{\Omega}\left|u_{i}\right|^{p} \mathrm{~d} x-\frac{1}{p^{\prime} \varepsilon^{p^{\prime}}} \int_{\Omega}|f|^{p^{\prime}} \mathrm{d} x
\end{aligned}
$$

Then 


$$
\begin{aligned}
m+1+\frac{1}{p^{\prime} \varepsilon^{p^{\prime}}} \int_{\Omega}|f|^{p^{\prime}} \mathrm{d} x & \geq \frac{1}{2 p}\left(\int_{\Omega}\left|\nabla u_{i}\right|^{p} \mathrm{~d} x+\beta \int_{\partial \Omega}\left|u_{i}\right|^{p} \mathrm{~d} \mathcal{H}^{n-1}(x)\right)-\frac{\varepsilon^{p}}{p} \int_{\Omega}\left|u_{i}\right|^{p} \mathrm{~d} x \\
& +\frac{1}{2 p}\left(\int_{\Omega}\left|\nabla u_{i}\right|^{p} \mathrm{~d} x+\beta \int_{\partial \Omega}\left|u_{i}\right|^{p} \mathrm{~d} \mathcal{H}^{n-1}(x)\right) \\
& \geq \frac{1}{2 p} \int_{\Omega}\left|\nabla u_{i}\right|^{p} \mathrm{~d} x+\left(\frac{\lambda_{1, \beta}(\Omega)-2 \varepsilon^{p}}{2 p}\right) \int_{\Omega}\left|u_{i}\right|^{p} \mathrm{~d} x .
\end{aligned}
$$

Then, the minimizing sequence $\left\{u_{i}\right\}$ is bounded in $W^{1, p}(\Omega)$, so there exists a subsequence $\left\{u_{i_{k}}\right\}$ weakly converging in $W^{1, p}(\Omega)$ and strongly in $L^{p}(\Omega)$ to a function $u$. Let us show that $u$ is the minimum.

The function $t^{p}$ is strictly convex for $p>1$, so

$$
\begin{gathered}
\left|u_{i_{k}}\right|^{p} \geq|u|^{p}+p|u|^{p-2} u\left(u_{i_{k}}-u\right) \\
\left|\nabla u_{i_{k}}\right|^{p} \geq|\nabla u|^{p}+p|\nabla u|^{p-2} \nabla u\left(\nabla u_{i_{k}}-\nabla u\right)
\end{gathered}
$$

Putting (8) e (9) in $\mathfrak{F}\left(u_{i_{k}}\right)$, we obtain

$$
\begin{aligned}
\int_{\Omega} f u_{i_{k}} \mathrm{~d} x+\mathfrak{F}\left(u_{i_{k}}\right) & \geq \frac{1}{p} \int_{\Omega}|\nabla u|^{p} \mathrm{~d} x+\int_{\Omega}|\nabla u|^{p-2} \nabla u\left(\nabla u_{i_{k}}-\nabla u\right) \\
& +\frac{\beta}{p} \int_{\partial \Omega}|u|^{p} \mathrm{~d} \mathcal{H}^{n-1}(x)+\beta \int_{\partial \Omega}|u|^{p-2} u\left(u_{i_{k}}-u\right) \mathrm{d} \mathcal{H}^{n-1}
\end{aligned}
$$

Passing to the limit for $k \rightarrow \infty$, by the weak convergence of $\left\{u_{i_{k}}\right\}$ the integral over $\Omega$ on the right-hand side goes to 0 . The integral over $\partial \Omega$ goes to 0 as well. Indeed, the space $W^{1, p}(\Omega)$ is compactly embedded in $L^{p}(\partial \Omega)$ (for more details, see [9] 2.5), and $u_{i_{k}}-u \rightarrow 0$ in $L^{p}(\partial \Omega)$. So, we obtain

$$
m \geq \mathfrak{F}(u) .
$$

This ensures us that $u$ is the minimum of the functional.

The uniqueness of the minimum follows from the fact that $\mathfrak{F}(u)$ is the sum of a strictly convex part and a linear part.

We observe that the solutions $u$ and $v$ to (1) and (3), respectively, are both $p$-superharmonic and then, by the strong maximum principle in [13], it follows that they achieve their minima on the boundary. Denoting by $u_{m}$ and $v_{m}$ the minimum of $u$ and $v$, respectively, thanks to the positiveness of $\beta$ and the Robin boundary conditions, we have that $u_{m} \geq 0$ and $v_{m} \geq 0$. Hence, $u$ and $v$ are strictly positive in the interior of $\Omega$. Moreover, we can observe that

$$
u_{m}=\min _{\Omega} u \leq \min _{\Omega^{\sharp}} v=v_{m},
$$

in fact, if we consider 


$$
\begin{aligned}
v_{m}^{p-1} \operatorname{Per}\left(\Omega^{\sharp}\right) & =\int_{\partial \Omega^{\sharp}} v(x)^{p-1} \mathrm{~d} \mathcal{H}^{n-1}(x)=\frac{1}{\beta} \int_{\Omega^{\sharp}} f^{\sharp} \mathrm{d} x=\frac{1}{\beta} \int_{\Omega} f \mathrm{~d} x \\
& =\int_{\partial \Omega} u(x)^{p-1} \mathrm{~d} \mathcal{H}^{n-1}(x) \\
& \geq u_{m}^{p-1} \operatorname{Per}(\Omega) \geq|u|_{m}^{p-1} \operatorname{Per}\left(\Omega^{\sharp}\right) .
\end{aligned}
$$

A consequence of (10) that will be used in what follows is that

$$
\mu(t) \leq \phi(t)=|\Omega| \quad \forall t \leq v_{m} .
$$

\subsection{Useful lemmas}

Let $u$ be the solution to (1). For $t \geq 0$, we denote by

$$
U_{t}=\{x \in \Omega: u(x)>t\} \quad \partial U_{t}^{\mathrm{int}}=\partial U_{t} \cap \Omega, \quad \partial U_{t}^{\mathrm{ext}}=\partial U_{t} \cap \partial \Omega,
$$

and by

$$
\mu(t)=\left|U_{t}\right| \quad P_{u}(t)=\operatorname{Per}\left(U_{t}\right)
$$

where $|\cdot|$ is the Lebesgue measure on $\mathbb{R}^{n}$ and $\operatorname{Per}(\cdot)$ is the perimeter.

If $v$ is the solution to (3), using the same notations, we set

$$
V_{t}=\left\{x \in \Omega^{\sharp}: v(x)>t\right\}, \quad \phi(t)=\left|V_{t}\right|, \quad P_{v}(t)=\operatorname{Per}\left(V_{t}\right) .
$$

Because of the invariance of the $p$-Laplacian and of the Schwarz rearrangement of $f$ by rotation, there exists a radial solution to (3) and, by uniqueness of solutions, this solution is $v$.

Since $v$ is radial, positive and decreasing along the radius, then for $0 \leq t \leq v_{m}, V_{t}=\Omega^{\sharp}$, while for $v_{m}<t<\max _{\Omega^{\sharp}} v, V_{t}$ is a ball, concentric to $\Omega^{\sharp}$ and strictly contained in it.

Lemma 2.1 (Gronwall) Let $\xi(t):\left[\tau_{0},+\infty[\rightarrow \mathbb{R}\right.$ be a continuous and differentiable function satisfying, for some non-negative constant $C$, the following differential inequality

$$
\tau \xi^{\prime}(\tau) \leq(p-1) \xi(\tau)+C \quad \forall \tau \geq \tau_{0}>0 .
$$

Then, we have

(i) $\xi(\tau) \leq\left(\xi\left(\tau_{0}\right)+\frac{C}{p-1}\right)\left(\frac{\tau}{\tau_{0}}\right)^{p-1}-\frac{C}{p-1} \quad \forall \tau \geq \tau_{0}$;

(ii) $\xi^{\prime}(\tau) \leq\left(\frac{(p-1) \xi\left(\tau_{0}\right)+C}{\tau_{0}}\right)\left(\frac{\tau}{\tau_{0}}\right)^{p-2} \quad \forall \tau \geq \tau_{0}$.

Proof Dividing both sides of the differential inequality by $\tau^{p}$, we obtain 


$$
\left(\frac{\xi^{\prime}(\tau)}{\tau^{p-1}}-(p-1) \frac{\xi(\tau)}{\tau^{p}}\right)=\left(\frac{\xi(\tau)}{\tau^{p-1}}\right)^{\prime} \leq \frac{C}{\tau^{p}}
$$

Now, we integrate from $\tau_{0}$ to $\tau$ and we obtain

$$
\begin{aligned}
\int_{\tau_{0}}^{\tau}\left(\frac{\xi(\tau)}{\tau^{p-1}}\right)^{\prime} \mathrm{d} \tau & \leq \int_{\tau_{0}}^{\tau} \frac{C}{\tau^{p}} \mathrm{~d} \tau \\
\Longrightarrow \xi(\tau) & \leq\left(\xi\left(\tau_{0}\right)+\frac{C}{p-1}\right)\left(\frac{\tau}{\tau_{0}}\right)^{p-1}-\frac{C}{p-1},
\end{aligned}
$$

which gives (i).

In order to obtain (ii), we just take into account (i) in the differential inequality.

Lemma 2.2 Let $u$ and $v$ be solutions to (1) and (3), respectively. Then for almost every $t>0$, we have

$$
\gamma_{n} \mu(t)^{\left(1-\frac{1}{n}\right) \frac{p}{p-1}} \leq\left(\int_{0}^{\mu(t)} f^{*}(s) \mathrm{d} s\right)^{\frac{1}{p-1}}\left(-\mu^{\prime}(t)+\frac{1}{\beta^{\frac{1}{p-1}}} \int_{\partial U_{t}^{\text {ext }}} \frac{1}{u} \mathrm{~d} \mathcal{H}^{n-1}(x)\right),
$$

where $\gamma_{n}=\left(n \omega_{n}^{1 / n}\right)^{\frac{p}{p-1}}$.

And

$$
\gamma_{n} \phi(t)^{\left(1-\frac{1}{n}\right) \frac{p}{p-1}}=\left(\int_{0}^{\phi(t)} f^{*}(s) \mathrm{d} s\right)^{\frac{1}{p-1}}\left(-\phi^{\prime}(t)+\frac{1}{\beta^{\frac{1}{p-1}}} \int_{\partial V_{t}^{\text {ext }}} \frac{1}{v} \mathrm{~d} \mathcal{H}^{n-1}(x)\right) .
$$

Proof Let $t>0$ e $h>0$, we choose the test function

$$
\varphi(x)= \begin{cases}0 & \text { if } u<t \\ u-t & \text { if } t<u<t+h \\ h & \text { if } u>t+h .\end{cases}
$$

Then,

$$
\begin{aligned}
\int_{U_{t} \backslash U_{t+h}}|\nabla u|^{p} \mathrm{~d} x & +\beta h \int_{\partial U_{t+h}^{e x t}} u^{p-1} \mathrm{~d} \mathcal{H}^{n-1}(x)+\beta \int_{\partial U_{t}^{e x t} \backslash \partial U_{t+h}^{e x t}} u^{p-1}(u-t) \mathrm{d} \mathcal{H}^{n-1}(x) \\
& =\int_{U_{t} \backslash U_{t+h}} f(u-t) \mathrm{d} x+h \int_{U_{t+h}} f \mathrm{~d} x .
\end{aligned}
$$

Dividing by $h$, using coarea formula and letting $h$ go to 0 , we have that for a. e. $t>0$

$$
\int_{\partial U_{t}} g(x) \mathrm{d} \mathcal{H}^{n-1}(x)=\int_{U_{t}} f \mathrm{~d} x
$$

where 


$$
g(x)= \begin{cases}|\nabla u|^{p-1} & \text { if } x \in \partial U_{t}^{i n t} \\ \beta u^{p-1} & \text { if } x \in \partial U_{t}^{e x t}\end{cases}
$$

So, using the isoperimetric inequality, for a. e. $t>0$, we have

$$
\begin{aligned}
& n \omega_{n}^{1 / n} \mu(t)^{\left(1-\frac{1}{n}\right)} \leq P_{u}(t)=\int_{\partial U_{t}} \mathrm{~d} \mathcal{H}^{n-1}(x) \leq\left(\int_{\partial U_{t}} g \mathrm{~d} \mathcal{H}^{n-1}(x)\right)^{\frac{1}{p}}\left(\int_{\partial U_{t}} \frac{1}{g^{\frac{1}{p-1}}} \mathrm{~d} \mathcal{H}^{n-1}(x)\right)^{1-\frac{1}{p}} \\
& =\left(\int_{\partial U_{t}} g \mathrm{~d} \mathcal{H}^{n-1}(x)\right)^{\frac{1}{p}}\left(\int_{\partial U_{t}^{i n t}} \frac{1}{|\nabla u|} \mathrm{d} \mathcal{H}^{n-1}(x)+\frac{1}{\beta^{\frac{1}{p-1}}} \int_{\partial U_{t}^{e x t}} \frac{1}{u} \mathrm{~d} \mathcal{H}^{n-1}(x)\right)^{1-\frac{1}{p}} \\
& \leq\left(\int_{0}^{\mu(t)} f^{*}(s) d s\right)^{\frac{1}{p}}\left(-\mu^{\prime}(t)+\frac{1}{\beta^{\frac{1}{p-1}}} \int_{\partial U_{t}^{e x t}} \frac{1}{u} \mathrm{~d} \mathcal{H}^{n-1}(x)\right)^{1-\frac{1}{p}} \quad t \in\left[0, \max _{\Omega} u\right),
\end{aligned}
$$

Then (12) follows. We notice that if $v$ is the solution to (3), than all the inequalities are verified as equalities, so we have (13).

Lemma 2.3 For all $\tau \geq v_{m}$, we have

$$
\int_{0}^{\tau} t^{p-1}\left(\int_{\partial U_{t}^{e x t}} \frac{1}{u(x)} \mathrm{d} \mathcal{H}^{n-1}(x)\right) \mathrm{d} t \leq \frac{1}{p \beta} \int_{0}^{|\Omega|} f^{*}(s) \mathrm{d} s .
$$

Moreover,

$$
\int_{0}^{\tau} t^{p-1}\left(\int_{\partial V_{t} \cap \partial \Omega^{\sharp}} \frac{1}{v(x)} \mathrm{d} \mathcal{H}^{n-1}(x)\right) \mathrm{d} t=\frac{1}{p \beta} \int_{0}^{|\Omega|} f^{*}(s) \mathrm{d} s,
$$

Proof If we integrate the quantity

$$
t^{p-1}\left(\int_{\partial U_{t}^{\text {ext }}} \frac{1}{u(x)} \mathrm{d} \mathcal{H}^{n-1}(x)\right)
$$

from 0 to $+\infty$, by Fubini theorem, we obtain

$$
\begin{aligned}
\int_{0}^{\infty} \tau^{p-1}\left(\int_{\partial U_{\tau}^{\text {ext }}} \frac{1}{u(x)} \mathrm{d} \mathcal{H}^{n-1}(x)\right) \mathrm{d} \tau & =\int_{\partial \Omega}\left(\int_{0}^{u(x)} \frac{\tau^{p-1}}{u(x)} \mathrm{d} \tau\right) \mathrm{d} \mathcal{H}^{n-1}(x) \\
& =\frac{1}{p} \int_{\partial \Omega} u(x)^{p-1} \mathrm{~d} \mathcal{H}^{n-1}(x) \\
& =\frac{1}{p \beta} \int_{0}^{|\Omega|} f^{*}(s) \mathrm{d} s,
\end{aligned}
$$

where the last equality follows from the fact that $u$ solves (1).

Analogously

$$
\int_{0}^{\infty} \tau^{p-1}\left(\int_{\partial V_{\tau} \cap \partial \Omega^{\sharp}} \frac{1}{v(x)} \mathrm{d} \mathcal{H}^{n-1}(x)\right) \mathrm{d} \tau=\frac{1}{p \beta} \int_{0}^{|\Omega|} f^{*}(s) \mathrm{d} s .
$$

Since $u$ is positive, we obtain, $\forall t \geq 0$, 


$$
\int_{0}^{t} \tau^{p-1}\left(\int_{\partial U_{\tau}^{e x t}} \frac{1}{u(x)} \mathrm{d} \mathcal{H}^{n-1}(x)\right) \mathrm{d} \tau \leq \frac{1}{p \beta} \int_{0}^{|\Omega|} f^{*}(s) \mathrm{d} s,
$$

on the other hand, since $\partial V_{t} \cap \partial \Omega^{\sharp}$ is empty for $t \geq v_{m}$, we have

$$
\int_{0}^{t} \tau^{p-1}\left(\int_{\partial V_{\tau} \cap \partial \Omega^{\sharp}} \frac{1}{v(x)} \mathrm{d} \mathcal{H}^{n-1}(x)\right) \mathrm{d} \tau=\frac{1}{p \beta} \int_{0}^{|\Omega|} f^{*}(s) \mathrm{d} s .
$$

and the proof of Lemma 2.3 is complete.

Remark 2.1 It can be observed that, since $\partial V_{t} \cap \partial \Omega^{\sharp}$ is empty for $t \geq v_{m}$ and $\phi(t)=|\Omega|$ for $t \leq v_{m}$, for all $\delta>0$ and for all $t$, we have

$$
\begin{aligned}
\int_{0}^{t} & \tau^{p-1} \phi(\tau)^{\delta}\left(\int_{\partial V_{\tau} \cap \partial \Omega^{\sharp}} \frac{1}{v(x)} \mathrm{d} \mathcal{H}^{n-1}(x)\right) \mathrm{d} \tau \\
= & \int_{0}^{v_{m}} \tau^{p-1} \phi(\tau)^{\delta}\left(\int_{\partial V_{\tau} \cap \partial \Omega^{\sharp}} \frac{1}{v(x)} \mathrm{d} \mathcal{H}^{n-1}(x)\right) \mathrm{d} \tau \\
= & \int_{0}^{+\infty} \tau^{p-1} \phi(\tau)^{\delta}\left(\int_{\partial V_{\tau} \cap \partial \Omega^{\sharp}} \frac{1}{v(x)} \mathrm{d} \mathcal{H}^{n-1}(x)\right) \mathrm{d} \tau=\frac{|\Omega|^{\delta}}{p \beta} \int_{0}^{|\Omega|} f^{*}(s) \mathrm{d} s .
\end{aligned}
$$

\section{Main results}

Now, we prove Theorems 1.1 and 1.2.

Proof of Theorem 1.1 Let $0<k \leq \frac{n(p-1)}{p(n-1)}$, so $\delta=\frac{1}{k}-\frac{(n-1) p}{n(p-1)}$ is positive.

Multiplying (12) by $t^{p-1} \mu(t)^{\delta}$ and integrating from 0 to $\tau \geq v_{m}$, by the previous Lemma, we obtain

$$
\begin{aligned}
\int_{0}^{\tau} \gamma_{n} t^{p-1} \mu(t)^{\frac{1}{k}} \mathrm{~d} t & \leq \int_{0}^{\tau}\left(-\mu^{\prime}(t)\right) t^{p-1} \mu(t)^{\delta}\left(\int_{0}^{\mu(t)} f^{*}(s) \mathrm{d} s\right)^{\frac{1}{p-1}} \mathrm{~d} t \\
& +\frac{|\Omega|^{\delta}}{p \beta^{\frac{p}{p-1}}}\left(\int_{0}^{|\Omega|} f^{*}(s) \mathrm{d} s\right)^{\frac{p}{p-1}} .
\end{aligned}
$$

Setting $F(l)=\int_{0}^{l} \omega^{\delta}\left(\int_{0}^{\omega} f^{*}(s) \mathrm{d} s\right)^{\frac{1}{p-1}} \mathrm{~d} \omega$, we can integrate by parts both sides of the last inequality, getting

$$
\begin{aligned}
\tau^{p-1}\left(\left(\int_{0}^{\tau} \gamma_{n} \mu(t)^{\frac{1}{k}} \mathrm{~d} t\right)+F(\mu(\tau))\right) & \leq(p-1) \int_{0}^{\tau} t^{p-2}\left(\left(\int_{0}^{t} \gamma_{n} \mu(s)^{\frac{1}{k}} \mathrm{~d} s\right)+F(\mu(t))\right) \mathrm{d} t \\
& +\frac{|\Omega|^{\delta}}{p \beta^{\frac{p}{p-1}}}\left(\int_{0}^{|\Omega|} f^{*}(s) \mathrm{d} s\right)^{\frac{p}{p-1}} .
\end{aligned}
$$


Setting $\xi(\tau)=\int_{0}^{\tau} t^{p-2}\left(\int_{0}^{t} \gamma_{n} \mu(s)^{\frac{1}{k}} \mathrm{~d} s+F(\mu(t))\right) \mathrm{d} t$ and $C=\frac{|\Omega|^{\delta}}{p \beta^{\frac{p}{p-1}}}\left(\int_{0}^{|\Omega|} f^{*}(s) \mathrm{d} s\right)^{\frac{p}{p-1}}$, we are in the hypothesis of Lemma 2.1 (Gronwall), namely

$$
\tau \xi^{\prime}(\tau) \leq(p-1) \xi(\tau)+C
$$

so, choosing $\tau_{0}=v_{m}$, we have

$$
\tau^{p-2}\left(\int_{0}^{\tau} \gamma_{n} \mu(s)^{\frac{1}{k}} \mathrm{~d} s+F(\mu(\tau))\right) \leq\left(\frac{(p-1) \xi\left(v_{m}\right)+C}{v_{m}}\right)\left(\frac{\tau}{v_{m}}\right)^{p-2},
$$

where

$$
\xi\left(v_{m}\right)=\int_{0}^{v_{m}} t^{p-2}\left(\int_{0}^{t} \gamma_{n} \mu(s)^{\frac{1}{k}} \mathrm{~d} s+F(\mu(t))\right) \mathrm{d} t .
$$

The previous inequality becomes an equality if we replace $\mu(t)$ with $\phi(t)$. Since $\mu(t) \leq \phi(t)=|\Omega|, \quad \forall t \leq v_{m}$, and $F(l)$ is monotone, we obtain

$$
\int_{0}^{v_{m}} t^{p-2}\left(\int_{0}^{t} \gamma_{n} \mu(s)^{\frac{1}{k}} \mathrm{~d} s+F(\mu(t))\right) \mathrm{d} t \leq \int_{0}^{v_{m}} t^{p-2}\left(\int_{0}^{t} \gamma_{n} \phi(s)^{\frac{1}{k}} \mathrm{~d} s+F(\phi(t))\right) \mathrm{d} t,
$$

hence

$$
\int_{0}^{\tau} \gamma_{n} \mu(s)^{\frac{1}{k}} \mathrm{~d} s+F(\mu(\tau)) \leq \int_{0}^{\tau} \gamma_{n} \phi(s)^{\frac{1}{k}} \mathrm{~d} s+F(\phi(\tau)) .
$$

Passing to the limit as $\tau \rightarrow \infty$, we get

$$
\int_{0}^{\infty} \mu(t)^{\frac{1}{k}} \mathrm{~d} t \leq \int_{0}^{\infty} \phi(t)^{\frac{1}{k}} \mathrm{~d} t
$$

and hence

$$
\|u\|_{L^{k, 1}(\Omega)} \leq\|v\|_{L^{k, 1}\left(\Omega^{\sharp}\right)} \quad \forall 0<k \leq \frac{n(p-1)}{p(n-1)} .
$$

To prove the inequality (5), it is enough to show that

$$
\int_{0}^{\infty} t^{p-1} \mu(t)^{\frac{1}{k}} \mathrm{~d} t \leq \int_{0}^{\infty} t^{p-1} \phi(t)^{\frac{1}{k}} \mathrm{~d} t
$$

Let us consider equation (16), let us integrate by parts the first term on the right-hand side from 0 to $\tau$ and then let us pass to the limit as $\tau \rightarrow \infty$, we have

$$
\int_{0}^{\infty} \gamma_{n} t^{p-1} \mu(t)^{\frac{1}{k}} \mathrm{~d} t \leq(p-1) \int_{0}^{\infty} t^{p-2} F(\mu(t)) \mathrm{d}+\frac{|\Omega|^{\delta}}{p \beta^{1+\frac{1}{p-1}}}\left(\int_{0}^{|\Omega|} f^{*}(s) \mathrm{d} s\right)^{\frac{p}{p-1}} .
$$

Therefore, if we show that 


$$
\int_{0}^{\infty} t^{p-2} F(\mu(t)) \mathrm{d} t \leq \int_{0}^{\infty} t^{p-2} F(\phi(t)) \mathrm{d} t
$$

we obtain the (17). To this aim, we multiply (12) by $t^{p-1} F(\mu(t)) \mu(t)^{-\frac{(n-1) p}{n(p-1)}}$ and integrate. First, we observe that, by the choice $k \leq \frac{n(p-1)}{(n-2) p+n}$, it follows that the function $h(l)=F(l) l^{-\frac{(n-1) p}{n(p-1)}}$ is non decreasing. Hence, we obtain

$$
\begin{aligned}
\int_{0}^{\tau} \gamma_{n} t^{p-1} F(\mu(t)) \mathrm{d} t & \leq \int_{0}^{\tau}\left(-\mu^{\prime}(t)\right) t^{p-1} \mu(t)^{-\frac{(n-1) p}{n(p-1)}} F(\mu(t))\left(\int_{0}^{\mu(t)} f^{*}(s) \mathrm{d} s\right)^{\frac{1}{p-1}} \mathrm{~d} t \\
& +F(|\Omega|) \frac{|\Omega|^{-\frac{(n-1) p}{n(p-1)}}}{p \beta^{\frac{p}{p-1}}}\left(\int_{0}^{|\Omega|} f^{*}(s) \mathrm{d} s\right)^{\frac{p}{p-1}} .
\end{aligned}
$$

If we integrate by parts both sides of the last expression and set

$$
C=F(|\Omega|) \frac{|\Omega|^{-\frac{p(n-1)}{n(p-1)}}}{p \beta^{\frac{p}{p-1}}}\left(\int_{0}^{|\Omega|} f^{*}(s) \mathrm{d} s\right)^{\frac{p}{p-1}}
$$

we obtain

$$
\tau \int_{0}^{\tau} \gamma_{n} t^{p-2} F(\mu(t)) \mathrm{d} t+\tau H_{\mu}(\tau) \leq \int_{0}^{\tau} \int_{0}^{t} r^{p-2} F(\mu(r)) \mathrm{d} r \mathrm{~d} t+\int_{0}^{\tau} H_{\mu}(t) \mathrm{d} t+C
$$

where

$$
H_{\mu}(\tau)=-\int_{\tau}^{+\infty} t^{p-2} \mu(t)^{-\frac{p(n-1)}{n(p-1)}} F(\mu(t))\left(\int_{0}^{\mu(t)} f^{*}(s) \mathrm{d} s\right)^{\frac{1}{p-1}} \mathrm{~d} \mu(t) .
$$

Setting

$$
\xi(\tau)=\int_{0}^{\tau} \int_{0}^{t} \gamma_{n} r^{p-2} F(\mu(r)) \mathrm{d} r+\int_{0}^{t} H_{\mu}(t) \mathrm{d} t
$$

then (19) becomes

$$
\tau \xi^{\prime}(\tau) \leq \xi(\tau)+C
$$

So lemma 2.1, with $\tau_{0}=v_{m}$, gives

$$
\int_{0}^{\tau} \gamma_{n} t^{p-2} F(\mu(t)) \mathrm{d} t+H_{\mu}(\tau) \leq\left(\frac{(p-1) \int_{0}^{v_{m}} t^{p-2} F\left(\mu(t) \mathrm{d} t+H_{\mu}\left(v_{m}\right)+C\right.}{v_{m}}\right)\left(\frac{\tau}{v_{m}}\right)^{p-2}
$$

Of course, the inequality holds as an equality if we replace $\mu(t)$ with $\phi(t)$, so we get, keeping in mind that $\mu(t) \leq \phi(t)=|\Omega|$ for $t \leq v_{m}$,

$$
\int_{0}^{\tau} \gamma_{n} t^{p-2} F\left(\mu(t) \mathrm{d} t+H_{\mu}(\tau) \leq \int_{0}^{\tau} \gamma_{n} F(\phi(t)) \mathrm{d} t+H_{\phi}(\tau)\right.
$$


Letting $\tau \rightarrow \infty$, one has

$$
\int_{0}^{\infty} t^{p-2} F(\mu(t)) \mathrm{d} t \leq \int_{0}^{\infty} t^{p-2} F(\phi(t)) \mathrm{d} t
$$

as $H_{\mu}(\tau), H_{\phi}(\tau) \rightarrow 0$. This proves (18), and hence (5).

The fact that both $H_{\mu}$ and $H_{\phi}$ go to 0 as $\tau$ goes to infinity can be easily deduced distinguishing the cases.

- If $p \geq 2$

$$
\begin{aligned}
t^{p-2} \mu(t) & =\int_{u>t} t^{p-2} \mathrm{~d} x \leq \int_{u>t} u^{p-2} \mathrm{~d} x \leq\|u\|_{L^{p}}^{p-2} \mu(t)^{\frac{2}{p}} \\
\Rightarrow\left|H_{\mu}(\tau)\right| & =\int_{\tau}^{+\infty} t^{p-2} F(\mu(t)) \mu(t)^{-\frac{p(n-1)}{n(p-1)}}\left(\int_{0}^{\mu(t)} f^{*}(s) \mathrm{d} s\right)\left(-\mu^{\prime}(t)\right) \mathrm{d} t \\
& \leq\left(\int_{0}^{|\Omega|} f^{*}(s) \mathrm{d} s\right)\|u\|_{L^{p}}^{p-2} \int_{\tau}^{+\infty} F(\mu(t)) \mu(t)^{\frac{2}{p}-\frac{p(n-1)}{n(p-1)}-1}\left(-\mu^{\prime}(t)\right) \mathrm{d} t \stackrel{\tau \rightarrow+\infty}{\longrightarrow} 0 .
\end{aligned}
$$

- If $p<2$

$$
\begin{aligned}
\left|H_{\mu}(\tau)\right| & =\int_{\tau}^{+\infty} t^{p-2} F(\mu(t)) \mu(t)^{-\frac{p(n-1)}{n(p-1)}}\left(\int_{0}^{\mu(t)} f^{*}(s) s\right)\left(-\mu^{\prime}(t)\right) \mathrm{d} t \\
& \leq \tau^{p-2} \int_{\tau}^{+\infty} F(\mu(t)) \mu(t)^{-\frac{p(n-1)}{n(p-1)}}\left(\int_{0}^{\mu(t)} f^{*}(s) s\right)\left(-\mu^{\prime}(t)\right) \mathrm{d} t \stackrel{\tau \rightarrow+\infty}{\longrightarrow} 0 .
\end{aligned}
$$

and analogously for $H_{\phi}$, which concludes the proof.

Proof of Theorem 1.2 (i) Firstly, we observe that $\int_{0}^{\mu(t)} f^{*}(s) \mathrm{d} s=\mu(t)$, so (12) becomes

$$
\gamma_{n} \mu(t)^{\left(1-\frac{1}{n}-\frac{1}{p}\right) \frac{p}{p-1}} \leq-\mu^{\prime}(t)+\frac{1}{\beta^{\frac{1}{p-1}}} \int_{\partial U_{t}^{\text {ext }}} \frac{1}{u} \mathrm{~d} \mathcal{H}^{n-1}(x) .
$$

Let us multiply both sides by $t^{p-1} \mu(t)^{\delta}$, where $\delta=-\left(1-\frac{1}{n}-\frac{1}{p}\right) \frac{p}{p-1}$. We point out that $\delta \geq 0$ for $p \leq \frac{n}{n-1}$. Hence, integrating from 0 to $\tau \geq v_{m}$, we have

$$
\begin{aligned}
\int_{0}^{\tau} \gamma_{n} t^{p-1} & \leq \int_{0}^{\tau} t^{p-1} \mu(t)^{\delta}\left(-\mu^{\prime}(t)\right) \mathrm{d} t+\frac{1}{\beta^{\frac{1}{p-1}}} \int_{0}^{\tau} t^{p-1} \mu(t)^{\delta} \int_{\partial U_{t}^{\text {ext }}} \frac{1}{u} \mathrm{~d} \mathcal{H}^{n-1}(x) \\
& \leq \int_{0}^{\tau} t^{p-1} \mu(t)^{\delta}\left(-\mu^{\prime}(t)\right) \mathrm{d} t+\frac{|\Omega|^{\delta+1}}{p \beta^{\frac{p}{p-1}}}
\end{aligned}
$$

Taking into account Remark 2.1, if we replace $\mu(t)$ with $\phi(t)$ the previous inequality holds as equality.

Hence, we get

$$
\int_{0}^{\tau} t^{p-1} \mu(t)^{\delta}\left(-\mu^{\prime}(t)\right) \mathrm{d} t \geq \int_{0}^{\tau} t^{p-1} \phi(t)^{\delta}\left(-\phi^{\prime}(t)\right) \mathrm{d} t
$$


Then, an integration by parts gives

$$
-\tau^{p-1} \frac{\mu(\tau)^{\delta+1}}{\delta+1}+(p-1) \int_{0}^{\tau} t^{p-2} \frac{\mu(t)^{\delta+1}}{\delta+1} \mathrm{~d} t \geq-\tau^{p-1} \frac{\phi(\tau)^{\delta+1}}{\delta+1}+(p-1) \int_{0}^{\tau} t^{p-2} \frac{\phi(t)^{\delta+1}}{\delta+1} \mathrm{~d} t .
$$

Finally, using Gronwall's Lemma with the function $\xi(\tau)=\int_{0}^{\tau} s^{p-2}\left(\frac{\mu(s)^{\delta+1}-\phi(s)^{\delta+1}}{\delta+1}\right) \mathrm{d} s$ we obtain

$$
\tau^{p-2}\left(\frac{\mu^{\delta+1}(\tau)-\phi^{\delta+1}(\tau)}{\delta+1}\right) \leq(p-1) \frac{\tau^{p-2}}{v_{m}^{p-2}} \int_{0}^{v_{m}} s^{p-2}\left(\frac{\mu^{\delta+1}(s)-\phi^{\delta+1}(s)}{\delta+1}\right) \mathrm{d} s .
$$

The quantity on the right-hand side is non-positive, thanks to (10), so

$$
\mu(\tau) \leq \phi(\tau) \quad \forall \tau \geq v_{m} .
$$

and, remembering that,

$$
\mu(\tau) \leq \phi(\tau)=|\Omega| \quad \forall \tau \leq v_{m},
$$

we get the point-wise inequality of the functions.

(ii) Now, we want to show that

$$
\|u\|_{L^{k, 1}(\Omega)} \leq\|v\|_{L^{k, 1}\left(\Omega^{\sharp}\right)}
$$

so it is enough to show

$$
\int_{0}^{+\infty} \mu(t)^{\frac{1}{k}} \mathrm{~d} t \leq \int_{0}^{+\infty} \phi(t)^{\frac{1}{k}} \mathrm{~d} t
$$

We multiply (20) by $t^{p-1} \mu(t)^{\frac{1}{k}-\left(1-\frac{1}{n}-\frac{1}{p}\right) \frac{p}{p-1}}$ and integrate from 0 to $\tau \geq v_{m}$. Then using Lemma 2.3 and Remark 2.1, we obtain

$$
\int_{0}^{\tau} \gamma_{n} t^{p-1} \mu(t)^{\frac{1}{k}} \mathrm{~d} t \leq \int_{0}^{\tau} t^{p-1} \mu(t)^{\frac{1}{k}-\left(1-\frac{1}{n}-\frac{1}{p}\right) \frac{p}{p-1}}\left(-\mu^{\prime}(t)\right) \mathrm{d} t+\frac{|\Omega|^{\frac{1}{k}-\left(1-\frac{1}{n}-\frac{1}{p}\right) \frac{p}{p-1}+1}}{p \beta^{\frac{p}{p-1}}}
$$

and equality holds if we replace $\mu$ with $\phi$. In order to be shorter, we set

$$
\eta=\frac{1}{k}-\left(1-\frac{1}{n}-\frac{1}{p}\right) \frac{p}{p-1}, \quad C=\frac{|\Omega|^{\eta+1}}{p \beta^{\frac{p}{p-1}}} .
$$

We point out that (23) follows by (21) if $\eta \geq 0$, namely

$$
0<k \leq \frac{n(p-1)}{n(p-1)-p}
$$

With these notations and keeping in mind that $\mu$ is a non increasing function, we have from (23) taht 


$$
\int_{0}^{\tau} \gamma_{n} t^{p-1} \mu(t)^{\frac{1}{k}} \mathrm{~d} t \leq \int_{0}^{\tau}-t^{p-1} \mu(t)^{\eta} \mathrm{d} \mu(t)+C
$$

Let us set $G(\ell)=\int_{0}^{\ell} w^{\eta} d w=\frac{\ell^{\eta+1}}{\eta+1}$, let us integrate by parts both sides of (24) in order to obtain

$$
\begin{aligned}
& \gamma_{n} \tau^{p-1} \int_{0}^{\tau} \mu(t)^{\frac{1}{k}} \mathrm{~d} t+\tau^{p-1} G(\mu(\tau)) \\
& \quad \leq(p-1)\left[\int_{0}^{\tau} \gamma_{n} t^{p-2} \int_{0}^{t} \mu(r)^{\frac{1}{k}} \mathrm{~d} r \mathrm{~d} t+\int_{0}^{\tau} t^{p-2} G(\mu(t)) \mathrm{d} t\right]+C
\end{aligned}
$$

Setting

$$
\xi(\tau)=\int_{0}^{\tau}\left(\gamma_{n} t^{p-2} \int_{0}^{t} \mu(r)^{\frac{1}{k}} \mathrm{~d} r\right) \mathrm{d} t+\int_{0}^{\tau} t^{p-2} G(\mu(t)) \mathrm{d} t
$$

(25) reads as follows

$$
\tau \xi^{\prime}(\tau) \leq(p-1) \xi(\tau)+C
$$

Hence, using Gronwall's Lemma 2.1 with $\tau_{0}=v_{m}$, we get

$$
\gamma_{n} \tau^{p-2} \int_{0}^{\tau} \mu(t)^{\frac{1}{k}} \mathrm{~d} t+\tau^{p-2} G(\mu(\tau)) \leq\left(\frac{(p-1) \xi\left(v_{m}\right)+C}{v_{m}}\right)\left(\frac{\tau}{v_{m}}\right)^{p-2}
$$

where

$$
\xi\left(v_{m}\right)=\int_{0}^{v_{m}} \gamma_{n} t^{p-2} \int_{0}^{t} \mu(r)^{\frac{1}{k}} \mathrm{~d} r \mathrm{~d} t+\int_{0}^{v_{m}} t^{p-2} G(\mu(t)) \mathrm{d} t
$$

Again, if we replace $\mu$ with $\phi$, the previous inequality holds as an equality and $\xi\left(v_{m}\right)$ is less or equal than the same quantity obtained by replacing $\mu$ with $\phi$, as (10) holds. Keeping in mind (11), we have

$$
\tau^{p-2}\left(\gamma_{n} \int_{0}^{\tau} \mu(t)^{\frac{1}{k}} \mathrm{~d} t+G(\mu(\tau))\right) \leq \tau^{p-2}\left(\gamma_{n} \int_{0}^{\tau} \phi(t)^{\frac{1}{k}} \mathrm{~d} t+G(\phi(\tau))\right)
$$

Passing to the limit as $\tau \rightarrow+\infty$, we get

$$
\int_{0}^{+\infty} \mu(t)^{\frac{1}{k}} \mathrm{~d} t \leq \int_{0}^{+\infty} \phi(t)^{\frac{1}{k}} \mathrm{~d} t
$$

namely (22).

To conclude the proof, we have to show that

$$
\|u\|_{L^{p k, p}(\Omega)} \leq\|v\|_{L^{p k, p}\left(\Omega^{\sharp}\right)} \quad \forall 0<k \leq \frac{n(p-1)}{n(p-1)-p}
$$

that is to say 


$$
\int_{0}^{+\infty} t^{p-1} \mu(t)^{\frac{1}{k}} \mathrm{~d} t \leq \int_{0}^{+\infty} t^{p-1} \phi(t)^{\frac{1}{k}} \mathrm{~d} t
$$

We consider (24), pass to the limit as $\tau \rightarrow+\infty$ and integrate by parts the first term on the right-hand side

$$
\int_{0}^{+\infty} \gamma_{n} t^{p-1} \mu(t)^{\frac{1}{k}} \mathrm{~d} t \leq(p-1) \int_{0}^{+\infty} t^{p-2} G(\mu(t)) \mathrm{d} t+C .
$$

Hence, it is enough to show that

$$
\int_{0}^{+\infty} t^{p-2} G(\mu(t)) \mathrm{d} t \leq \int_{0}^{+\infty} t^{p-2} G(\phi(t)) \mathrm{d} t .
$$

To this aim, we multiply (20) by $t^{p-1} G(\mu(t)) \mu(t)^{-\left(1-\frac{1}{n}-\frac{1}{p}\right) \frac{p}{p-1}}$ and integrate from 0 to $\tau \geq v_{m}$

$$
\begin{aligned}
\int_{0}^{\tau} \gamma_{n} t^{p-1} G(\mu(t)) \mathrm{d} t & \leq \int_{0}^{\tau} t^{p-1} G(\mu(t)) \mu(t)^{-\left(1-\frac{1}{n}-\frac{1}{p}\right) \frac{p}{p-1}} \mathrm{~d} \mu(t) \\
& +\frac{1}{\beta^{\frac{1}{p-1}}} \int_{0}^{\tau} t^{p-1} G(\mu(t)) \mu(t)^{-\left(1-\frac{1}{n}-\frac{1}{p}\right) \frac{p}{p-1}}\left(\int_{\partial U_{t}^{e x t}} \frac{1}{u} \mathrm{~d} \mathcal{H}^{n-1}\right) \mathrm{d} t
\end{aligned}
$$

Since $k \leq \frac{n(p-1)}{n(p-1)-p}$, using Lemma 2.3 and the fact that the function $G(\ell) \ell^{-\left(1-\frac{1}{n}-\frac{1}{p}\right) \frac{p}{p-1}}$ is non-decreasing, we obtain

$$
\int_{0}^{\tau} \gamma_{n} t^{p-1} G(\mu(t)) \mathrm{d} t \leq \int_{0}^{\tau} t^{p-1} G(\mu(t)) \mu(t)^{-\left(1-\frac{1}{n}-\frac{1}{p}\right) \frac{p}{p-1}} \mathrm{~d} \mu(t)+C
$$

with

$$
C=\frac{1}{p \beta^{\frac{p}{p-1}}} G(|\Omega|)|\Omega|^{-\left(1-\frac{1}{n}-\frac{1}{p}\right) \frac{p}{p-1}+1}
$$

If we replace $\mu$ with $\phi$ the previous inequality holds as an equality, thanks to (2.1). Now, let us integrate by parts both sides of (26), obtaining

$$
\tau \int_{0}^{\tau} \gamma_{n} t^{p-2} G(\mu(t)) \mathrm{d} t+\tau H(\tau) \leq \int_{0}^{\tau} \int_{0}^{t} \gamma_{n} t^{p-2} G(\mu(r)) \mathrm{d} r \mathrm{~d} t+\int_{0}^{\tau} H_{\mu}(t) \mathrm{d} t+C
$$

where

$$
H_{\mu}(\tau)=-\int_{\tau}^{+\infty} t^{p-2} G(\mu(t)) \mu(t)^{-\left(1-\frac{1}{n}-\frac{1}{p}\right) \frac{p}{p-1}} \mathrm{~d} \mu(t)
$$

Setting

$$
\xi(\tau)=\int_{0}^{\tau} \int_{0}^{t} \gamma_{n} t^{p-2} G(\mu(r)) \mathrm{d} r \mathrm{~d} t+\int_{0}^{\tau} H_{\mu}(t) \mathrm{d} t
$$

the (27) reads as follows 


$$
\tau \xi^{\prime}(\tau) \leq \xi(\tau)+C
$$

Again, using Gronwall's Lemma 2.1, we get

$$
\int_{0}^{\tau} \gamma_{n} t^{p-2} G(\mu(t)) \mathrm{d} t+H_{\mu}(\tau) \leq\left(\frac{(p-1) \xi\left(v_{m}\right)+C}{v_{m}}\right)\left(\frac{\tau}{v_{m}}\right)^{p-2}
$$

with

$$
\xi\left(v_{m}\right)=\int_{0}^{v_{m}} \int_{0}^{t} \gamma_{n} t^{p-2} G(\mu(r)) \mathrm{d} r \mathrm{~d} t+\int_{0}^{v_{m}} H_{\mu}(t) \mathrm{d} t .
$$

Keeping in mind that for $\phi$ the previous inequalities hold as equality and the fact that $G$ is not decreasing, $\xi\left(v_{m}\right)$ is less or equal to the same quantity obtained by replacing $\mu$ with $\phi$. Hence, we obtain

$$
\int_{0}^{\tau} \gamma_{n} t^{p-2} G(\mu(t)) \mathrm{d} t+H_{\mu}(\tau) \leq \int_{0}^{\tau} \gamma_{n} t^{p-2} G(\phi(t)) \mathrm{d} t+H_{\phi}(\tau)
$$

and passing to the limit as $\tau \rightarrow+\infty$, we finally get

$$
\int_{0}^{+\infty} t^{p-2} G(\mu(t)) \mathrm{d} t \leq \int_{0}^{+\infty} t^{p-2} G(\phi(t)) \mathrm{d} t
$$

indeed, as in the proof of Theorem 1.1, $H_{\mu}(\tau)$ and $H_{\phi}(\tau)$ go to 0 as $\tau \rightarrow \infty$.

That concludes the proof.

Corollary 3.1 Let $u$ and $v$ be the solutions to (1) and (3), respectively. Then, if $p \geq n$, we have

$$
\|u\|_{L^{1}(\Omega)} \leq\|v\|_{L^{1}\left(\Omega^{\sharp}\right)} \quad \text { and } \quad\|u\|_{L^{p}(\Omega)} \leq\|v\|_{L^{p}\left(\Omega^{\sharp}\right)} .
$$

Moreover, in the case $f \equiv 1$, Theorem 1.2 gives

$$
\|u\|_{L^{1}(\Omega)} \leq\|v\|_{L^{1}\left(\Omega^{\sharp}\right)} \quad \text { and } \quad\|u\|_{L^{p}(\Omega)} \leq\|v\|_{L^{p}\left(\Omega^{\sharp}\right)} \quad \forall p>1
$$

and the point-wise comparison for $p \leq \frac{n}{n-1}$.

Proof If $p \geq n$ the upper bounds of $k$, in both cases (4) e (5), are greater than 1 and so we can choose $k=1$. The assertion follows from the fact that

$$
\|\cdot\|_{L^{p, p}(\Omega)}=\|\cdot\|_{L^{p}(\Omega)} .
$$

Analogously if $f \equiv 1$.

\section{Faber-Krahn inequality}

We recall that the first eigenvalue of $p$-Laplace operator with Robin boundary conditions is obtained as the minimum of the Rayleigh quotients, i.e., 


$$
\lambda_{1, \beta}(\Omega)=\min _{\substack{\omega \in W^{1, p}(\Omega) \\ \omega \neq 0}} \frac{\int_{\Omega}|\nabla \omega|^{p} \mathrm{~d} x+\beta \int_{\partial \Omega}|\omega|^{p} \mathrm{~d} \mathcal{H}^{n-1}(x)}{\int_{\Omega}|\omega|^{p} \mathrm{~d} x} .
$$

We can observe that if $u$ achieves the minimum of Rayleigh quotients, so does $|u|$. From this we have that $u$ in non-negative. Furthermore, we have if $u_{\lambda_{1, \beta}} \geq 0$, as a consequence of Harnack inequality, $u_{\lambda_{1, \beta}}>0$.

Another important thing is that the eigenvalue is simple. Indeed, as shown in [8], if $\Omega$ is smooth enough and $u$ and $v$ are to eigenfunctions referred to the first eigenvalue, we can choose as test function $\varphi_{1}=\frac{u^{p}-v^{p}}{u^{p-1}}$ in

$$
\int_{\Omega}|\nabla u|^{p-2} \nabla u \nabla \varphi_{1} \mathrm{~d} x+\beta \int_{\partial \Omega} u^{p-1} \varphi_{1} \mathrm{~d} \mathcal{H}^{n-1}(x)=\int_{\Omega} \lambda_{1, \beta} u^{p-1} \varphi_{1} \mathrm{~d} x
$$

and $\varphi_{2}=\frac{v^{p}-u^{p}}{v^{p-1}}$ in

$$
\int_{\Omega}|\nabla v|^{p-2} \nabla v \nabla \varphi_{2} \mathrm{~d} x+\beta \int_{\partial \Omega} v^{p-1} \varphi_{2} \mathrm{~d} \mathcal{H}^{n-1}(x)=\int_{\Omega} \lambda_{1, \beta} v^{p-1} \varphi_{2} \mathrm{~d} x .
$$

Summing the two equations, we have

$$
\begin{aligned}
0= & \int_{\Omega}\left\{1+(p-1)\left(\frac{v}{u}\right)^{p}\right\}|\nabla u|^{p}+\left\{1+(p-1)\left(\frac{u}{v}\right)^{p}\right\}|\nabla v|^{p} \\
& -\int_{\Omega} p\left(\frac{v}{u}\right)^{p-1}|\nabla u|^{p-2} \nabla u \nabla v+p\left(\frac{u}{v}\right)^{p-1}|\nabla v|^{p-2} \nabla v \nabla u \\
= & \int_{\Omega}\left(u^{p}-v^{p}\right)\left(|\nabla \log u|^{p}-|\nabla \log v|^{p}\right) \\
& -\int_{\Omega} p v^{p}|\nabla \log u|^{p-2}|\nabla \log u|(\nabla \log v-\nabla \log u) \\
& -\int_{\Omega} p u^{p}|\nabla \log v|^{p-2}|\nabla \log v|(\nabla \log u-\nabla \log v)
\end{aligned}
$$

Now, using the well known inequalities, which hold true for each $w_{1}$ and $w_{2} \in \mathbb{R}^{n}$,

$$
\begin{aligned}
& \left|w_{2}\right|^{p} \geq\left|w_{1}\right|^{p}+p\left|w_{1}\right|^{p-2} w_{1} \cdot\left(w_{2}-w_{1}\right)+\frac{\left|w_{2}-w_{1}\right|^{p}}{2^{p-1}-1} \quad \text { if } \quad p \geq 2 \\
& \left|w_{2}\right|^{p} \geq\left|w_{1}\right|^{p}+p\left|w_{1}\right|^{p-2} w_{1} \cdot\left(w_{2}-w_{1}\right)+C(p) \frac{\left|w_{2}-w_{1}\right|^{2}}{\left(\left|w_{1}\right|+\left|w_{2}\right|\right)^{2-p}} \quad \text { if } 1<p<2,
\end{aligned}
$$

if we consider the case $p \geq 2$, we choose $w_{2}=\nabla \log u$ and $w_{1}=\nabla \log v$, we obtain

$$
\frac{1}{2^{p-1}-1} \int_{\Omega}\left(\frac{1}{v^{p}}+\frac{1}{u^{p}}\right)|v \nabla u-u \nabla v|^{p}=0 .
$$

Hence, we obtain that $v \nabla u=u \nabla v$ a.e. in $\Omega$, and so there exists a constant $K$ for which $u=K v$. This means that $\lambda_{1, \beta}$ is simple.

For the proof of (29), we refer to [8]. 
The following corollary of Theorem 1.1 holds true, that is Faber-Krahn inequality.

Corollary 4.1 Let $u$ and $v$ be the solutions to (1) and (3), respectively. Then, if $p \geq n$, we have

$$
\lambda_{1, \beta}(\Omega) \geq \lambda_{1, \beta}\left(\Omega^{\sharp}\right)
$$

Proof Let $u$ an eigenfunction referred to the first eigenvalue of (1), then it solves

$$
\begin{cases}-\Delta_{p} u=\lambda_{1, \beta}(\Omega)|u|^{p-2} u & \text { in } \Omega \\ |\nabla u|^{p-2} \frac{\partial u}{\partial \nu}+\beta|u|^{p-2} u=0 & \text { on } \partial \Omega .\end{cases}
$$

Now, let $z$ be a solution to the following problem

$$
\begin{cases}-\Delta_{p} z=\lambda_{1, \beta}(\Omega)\left|u^{\sharp}\right|^{p-2} u^{\sharp} & \text { in } \Omega^{\sharp} \\ |\nabla z|^{p-2} \frac{\partial z}{\partial \nu}+\beta|z|^{p-2} z=0 & \text { on } \partial \Omega^{\sharp} .\end{cases}
$$

In that case, Corollary 3.1 gives

$$
\int_{\Omega}|u|^{p} \mathrm{~d} x=\int_{\Omega^{\sharp}}\left|u^{\sharp}\right|^{p} \mathrm{~d} x \leq \int_{\Omega^{\sharp}}|z|^{p} \mathrm{~d} x,
$$

and hence, by Hölder inequality

$$
\int_{\Omega^{\sharp}}\left(u^{\sharp}\right)^{p-2} u^{\sharp} z \mathrm{~d} x \leq\left(\int_{\Omega^{\sharp}}\left|u^{\sharp}\right|^{p} \mathrm{~d} x\right)^{\frac{p-1}{p}}\left(\int_{\Omega^{\sharp}} z^{p} \mathrm{~d} x\right)^{\frac{1}{p}} \leq \int_{\Omega^{\sharp}} z^{p} \mathrm{~d} x .
$$

Therefore, observing that we can write the eigenvalue $\lambda_{1, \beta}(\Omega)$ in the following way, we obtain

$$
\begin{aligned}
\lambda_{1, \beta}(\Omega) & =\frac{\int_{\Omega^{\sharp}}|\nabla z|^{p} \mathrm{~d} x+\beta \int_{\partial \Omega^{\sharp}} z^{p} \mathrm{~d} \mathcal{H}^{n-1}(x)}{\int_{\Omega^{\sharp}}\left(u^{\sharp}\right)^{p-2} u^{\sharp} z \mathrm{~d} x} \\
\geq & \frac{\int_{\Omega^{\sharp}}|\nabla z|^{p} \mathrm{~d} x+\beta \int_{\partial \Omega^{\sharp}} z^{p} \mathrm{~d} \mathcal{H}^{n-1}(x)}{\int_{\Omega^{\sharp}} z^{p} \mathrm{~d} x} \geq \lambda_{1, \beta}\left(\Omega^{\sharp}\right) .
\end{aligned}
$$

\section{Conclusions}

We have been able to extend the results obtained for the Laplacian to the $p$-Laplacian. Many problems remain open, such as 
Open Problem In the assumptions of Theorem 1.2, does the point-wise comparison hold also for $p>\frac{n}{n-1}$ ?

We have already observed in the Corollary 3.1 that if $p \geq n$ we have an estimate on the $L^{p}$ norms of $u$ and $v$. Can we generalize this estimate also for $q \neq p$ ? We know for sure that for $q=\infty$ this can't be done, as it can be seen in the following example.

Example 5.1 Let $\Omega \subseteq \mathbb{R}^{n}$ be the union of two disjoint balls, $B_{1}$ and $B_{r}$ with radii 1 and $r$, respectively. We choose $\beta<\left(\frac{n-1}{p-1}\right)^{p-1}$ with $p \neq n$, and we fix $f=1$ on $B_{1}$ and $f=0$ on $B_{r}$. Both $u$ and $v$ can be explicitly computed. We have $\|u\|_{\infty}-\|v\|_{\infty}=C r^{n}+o\left(r^{n}\right)$, where $C$ is a positive constant.

Proof We want an explicit expression of $u$ and $v$, respectively.

Starting from $u$, it is a solution to

$$
\begin{cases}-\operatorname{div}\left(|\nabla u|^{p-2} \nabla u\right)=f & \text { in } \Omega \\ |\nabla u|^{p-2} \frac{\partial u}{\partial \nu}+\beta|u|^{p-2} u=0 & \text { on } \partial \Omega .\end{cases}
$$

with $\left.f\right|_{B_{1}}=1$ and $\left.f\right|_{B_{r}}=0$.

It's clear that $\left.u\right|_{B_{r}}=0$ and $u(x)=u(|x|)$ it's radial on $B_{1}$.

So, the equation (1) becomes

$$
s^{n-1} \Delta_{p} u(s)=\frac{\mathrm{d}}{\mathrm{d} s}\left(s^{n-1}\left|u^{\prime}(s)\right|^{p-2} u^{\prime}(s)\right)
$$

and then

$$
\begin{aligned}
\frac{\mathrm{d}}{\mathrm{d} s}\left(s^{n-1}\left|u^{\prime}(s)\right|^{p-2} u^{\prime}(s)\right) & =s^{n-1} \Delta_{p} u(s)=-s^{n-1} \\
s^{n-1}\left|u^{\prime}(s)\right|^{p-2} u^{\prime}(s) & =-\frac{s^{n}}{n}+c .
\end{aligned}
$$

We set $c=0$, in order to have a $C^{1}$-solution.

$$
\left|u^{\prime}(s)\right|^{p-2} u^{\prime}(s)=-\frac{s}{n} \Longrightarrow u^{\prime}(s)=-\frac{s^{\frac{1}{p-1}}}{n^{\frac{1}{p-1}}} \quad \alpha=\frac{1}{p-1} .
$$

If we integrate, we obtain

$$
u(s)=-\frac{p-1}{n^{\alpha} p} s^{\frac{p}{p-1}}+A .
$$

The Robin boundary conditions become

$$
\left|u^{\prime}(1)\right|^{p-2} u^{\prime}(1)+\beta u(1)^{p-1}=0 \quad(u \geq 0),
$$

now we can compute the value of $A$ 


$$
-\frac{1}{n}+\beta\left(-\frac{p-1}{n^{\alpha} p}+A\right)^{p-1}=0 \Longrightarrow A=\frac{1}{(n \beta)^{\alpha}}+\frac{p-1}{n^{\alpha} p} .
$$

So

$$
u(s)=\frac{p-1}{n^{\alpha} p}\left(1-s^{\frac{p}{p-1}}\right)+\frac{1}{(n \beta)^{\alpha}} .
$$

As $u$ is decreasing, we have

$$
\|u\|_{\infty}=u(0)=\frac{p-1}{n^{\alpha} p}+\frac{1}{(n \beta)^{\alpha}} .
$$

Now, let us compute $v(s)$. We will do this firstly for $s \in(0,1)$, then for $s \in(1, \bar{r})$ where $\bar{r}=\left(1+r^{n}\right)^{\frac{1}{n}}$ is determined by the condition $|\Omega|=\left|\Omega^{\sharp}\right|$.

Let $s<1$

$$
\begin{aligned}
& \frac{\mathrm{d}}{\mathrm{d} s}\left(s^{n-1}\left|v^{\prime}(s)\right|^{p-2} v^{\prime}(s)\right)=-s^{n-1} \\
& \left|v^{\prime}(s)\right|^{p-2} v^{\prime}(s)=-\frac{s}{n} \Longrightarrow v^{\prime}(s)=-\frac{s^{\frac{1}{p-1}}}{n^{\alpha}} \\
& v(s)=-\frac{p-1}{n^{\alpha} p} s^{\frac{p}{p-1}}+B .
\end{aligned}
$$

Now, we can't determine $B$ as before, as $v$ is not identically 0 in the anulus $B_{\bar{r}} \backslash B_{1}$.

Let $s>1$ and $p \neq n$

$$
\begin{aligned}
& \frac{\mathrm{d}}{\mathrm{d} s}\left(s^{n-1}\left|v^{\prime}(s)\right|^{p-2} v^{\prime}(s)\right)=0 \\
& \left|v^{\prime}(s)\right|^{p-2} v^{\prime}(s)=\frac{C}{s^{n-1}}
\end{aligned}
$$

by imposing the continuity of the derivative for $s=1$, we obtain that $C=-1 / n$

$$
\begin{aligned}
v^{\prime}(s) & =-\frac{s^{-\frac{n-1}{p-1}}}{n^{\alpha}}, \\
v(s) & =-\frac{p-1}{n^{\alpha}(p-n)} s^{\frac{p-n}{p-1}}+D,
\end{aligned}
$$

and by Robin conditions

$$
\begin{aligned}
& \left|v^{\prime}(\bar{r})\right|^{p-2} v^{\prime}(\bar{r})+\beta v(\bar{r})^{p-1}=0, \\
& -\frac{\bar{r}^{-n-1}}{n}+\beta\left(-\frac{p-1}{n^{\alpha}(p-n)} \bar{r}^{\frac{p-n}{p-1}}+D\right)^{(p-1)}=0, \\
& D=\frac{1}{(n \beta)^{\alpha}} \bar{r}^{-\frac{n-1}{p-1}}+\frac{p-1}{n^{\alpha}(p-n)} \bar{r}^{\frac{p-n}{p-1}} .
\end{aligned}
$$

By imposing the continuity of $v$ for $s=1$, we have 


$$
B=\frac{p-1}{n^{\alpha} p}+\frac{\bar{r}^{-\frac{n-1}{p-1}}}{(n \beta)^{\alpha}}+\frac{p-1}{n^{\alpha}(p-n)}\left(\bar{r}^{\frac{p-n}{p-1}}-1\right)
$$

that is to say

$$
v(s)= \begin{cases}u(s)+\frac{1}{(n \beta)^{\alpha}}\left(\bar{r}^{-\frac{n-1}{p-1}}-1\right)+\frac{p-1}{n^{\alpha}(p-n)}\left(\bar{r}^{\frac{p-n}{p-1}}-1\right) & \text { if } s<1 \\ \frac{1}{(n \beta)^{\alpha}} \bar{r}^{-\frac{n-1}{p-1}}+\frac{p-1}{n^{\alpha}(p-n)}\left(\bar{r}^{\frac{p-n}{p-1}}-s^{\frac{p-n}{p-1}}\right) & \text { if } 1<s<\bar{r}\end{cases}
$$

For convenience's sake, we set $h=\frac{1}{(n \beta)^{\alpha}}\left(\bar{r}^{-\frac{n-1}{p-1}}-1\right)+\frac{p-1}{n^{\alpha}(p-n)}\left(\bar{r}^{\frac{p-n}{p-1}}-1\right)$.

So, we have

$$
\|v\|_{L^{\infty}\left(\Omega^{\sharp}\right)}=\|v\|_{L^{\infty}\left(B_{1}\right)}=\|u\|_{L^{\infty}(\Omega)}+h=u(0)+h
$$

By using Taylor expansion of the function $\left(1+r^{n}\right)^{\delta}$, we get

$$
h=\left(-\frac{1}{(n \beta)^{\alpha}} \frac{n-1}{n(p-1)}+\frac{1}{n^{\alpha+1}}\right) r^{n}+o\left(r^{n}\right),
$$

so, if we choose $\beta<\left(\frac{n-1}{p-1}\right)^{p-1}$, we get

$$
\|v\|_{L^{\infty}\left(\Omega^{\sharp}\right)}=\|u\|_{L^{\infty}(\Omega)}-C r^{n}+o\left(r^{n}\right) \text { where } C>0 .
$$

Next example 5.2 is a counterexample to the Corollary 3.1 in the case $n>p$.

Example 5.2 Let $\Omega \subseteq \mathbb{R}^{n}, p<n$ be the union of two disjoint balls $B_{1}$ and $B_{r}$ with radii 1 and $r$, respectively. We choose $\beta \leq\left(\frac{n-p}{p(p-1)}\right)^{p-1}$ and we fix $f=1$ on $B_{1}$ and $f=0$ on $B_{r}$. Both $u$ and $v$ can be explicitly computed. We have $\|u\|_{p}^{p}-\|v\|_{p}^{p}=C r^{n}+o\left(r^{n}\right)$, where $C$ is a positive constant.

Proof Let us consider the Taylor expansion of $(1+y)^{p}$, we get

$$
\|v\|_{L^{p}\left(B_{1}\right)}^{p}=\int_{B_{1}}(u+h)^{p}=\|u\|_{L^{p}\left(B_{1}\right)}^{p}+p\|u\|_{L^{p-1}\left(B_{1}\right)}^{p-1} h+o\left(r^{n}\right)
$$

Moreover,

$$
\|v\|_{L^{p}\left(B_{\bar{r}} \backslash B_{1}\right)}^{p}=\frac{\omega_{n}}{(n \beta)^{\alpha p}} r^{n}+o\left(r^{n}\right)
$$

as if $1<s<\bar{r}$

$$
\frac{1}{(n \beta)^{\alpha}} \bar{r}^{-\frac{n-1}{p-1}} \leq v(s) \leq \frac{1}{(n \beta)^{\alpha}} \bar{r}^{-\frac{n-1}{p-1}}+\frac{p-1}{n^{\alpha}(p-n)}\left(\bar{r}^{\frac{p-n}{p-1}}-1\right)
$$

thus

$$
v(s)=\frac{1}{(n \beta)^{\alpha}}+O\left(r^{n}\right)
$$


and by integration we obtain the value of the norm in $L^{p}\left(B_{\bar{r}} \backslash B_{1}\right)$.

So,

$$
\|v\|_{L^{p}\left(\Omega^{\sharp}\right)}^{p}=\|v\|_{L^{p}\left(B_{1}\right)}^{p}+\|v\|_{L^{p}\left(B_{\bar{r}} \backslash B_{1}\right)}^{p}=\|u\|_{L^{p}(\Omega)}^{p}+p\|u\|_{L^{p-1}\left(B_{1}\right)}^{p-1} h+\frac{\omega_{n}}{(n \beta)^{\alpha p}} r^{n}+o\left(r^{n}\right)
$$

and recalling that

$$
h=\left(-\frac{1}{(n \beta)^{\alpha}} \frac{n-1}{n(p-1)}+\frac{1}{n^{\alpha+1}}\right) r^{n}+o\left(r^{n}\right)
$$

we get

$$
\|v\|_{L^{p}\left(\Omega^{\sharp}\right)}^{p}=\|u\|_{L^{p}(\Omega)}^{p}+\left[p\|u\|_{L^{p-1}\left(B_{1}\right)}^{p-1}\left(-\frac{1}{(n \beta)^{\alpha}} \frac{n-1}{n(p-1)}+\frac{1}{n^{\alpha+1}}\right)+\frac{\omega_{n}}{(n \beta)^{\alpha p}}\right] r^{n}+o\left(r^{n}\right) .
$$

We have to understand whether

$$
p\|u\|_{L^{p-1}\left(B_{1}\right)}^{p-1}\left(-\frac{1}{(n \beta)^{\alpha}} \frac{n-1}{n(p-1)}+\frac{1}{n^{\alpha+1}}\right)+\frac{\omega_{n}}{(n \beta)^{\alpha p}}<0 .
$$

If we choose $\beta<\left(\frac{n-1}{p-1}\right)^{p-1}$, we have $-\frac{1}{(n \beta)^{\alpha}} \frac{n-1}{n(p-1)}+\frac{1}{n^{\alpha+1}}<0$. In order to have (30), we need

$$
\begin{aligned}
& \|u\|_{L^{p-1}\left(B_{1}\right)}^{p-1}>\frac{\omega_{n}}{(n \beta)^{\alpha p}}\left[\frac{n(p-1) n^{\alpha} \beta^{\alpha}}{p(n-1)-p \beta^{\alpha}(p-1)}\right] \\
& \|u\|_{L^{p-1}\left(B_{1}\right)}^{p-1}>\frac{\omega_{n}}{(n \beta)^{\alpha(p-1)}}\left[\frac{n(p-1)}{p(n-1)-p \beta^{\alpha}(p-1)}\right] .
\end{aligned}
$$

If we show that

$$
\left[\frac{n(p-1)}{p(n-1)-p \beta^{\alpha}(p-1)}\right] \leq 1
$$

then

$$
u(s)>\frac{1}{(n \beta)^{\alpha}} \Longrightarrow\|u\|_{L^{p-1}\left(B_{1}\right)}^{p-1}>\frac{\omega_{n}}{(n \beta)^{\alpha(p-1)}} .
$$

We just have to verify (31)

$$
\begin{aligned}
{\left[\frac{n(p-1)}{p(n-1)-p \beta^{\alpha}(p-1)}\right] \leq 1 } & \Longleftrightarrow n(p-1) \leq p(n-1)-p \beta^{\alpha}(p-1) \\
& \Longleftrightarrow p-n \leq-p(p-1) \beta^{\alpha}<0 \quad(\text { if and only if } p<n !) \\
& \Longleftrightarrow \beta \leq\left(\frac{n-p}{p(p-1)}\right)^{p-1}
\end{aligned}
$$


Funding Open access funding provided by Università degli Studi di Napoli Federico II within the CRUICARE Agreement.

Open Access This article is licensed under a Creative Commons Attribution 4.0 International License, which permits use, sharing, adaptation, distribution and reproduction in any medium or format, as long as you give appropriate credit to the original author(s) and the source, provide a link to the Creative Commons licence, and indicate if changes were made. The images or other third party material in this article are included in the article's Creative Commons licence, unless indicated otherwise in a credit line to the material. If material is not included in the article's Creative Commons licence and your intended use is not permitted by statutory regulation or exceeds the permitted use, you will need to obtain permission directly from the copyright holder. To view a copy of this licence, visit http://creativecommons.org/licenses/by/4.0/.

\section{References}

1. Alvino, A., Chiacchio, F., Nitsch, C., Trombetti, C.: Sharp estimates for solutions to elliptic problems with mixed boundary conditions. J. Math. Pures Appl. 152, 251-261 (2021)

2. Alvino, A., Lions, P.L., Trombetti, G.: Comparison results for elliptic and parabolic equations via Schwarz symmetrization. Ann. Inst. H. Poincaré Anal. Non Linéaire 7(2), 37-65 (1990)

3. Alvino, A., Nitsch, C., Trombetti, C.: A Talenti comparison result for solutions to elliptic problems with robin boundary conditions. to appear on Commun. Pure Appl. Math

4. Bucur, D., Daners, D.: An alternative approach to the Faber-Krahn inequality for Robin problems. Calc. Var. Partial Differ. Equ. 37(1-2), 75-86 (2010)

5. Bucur, D., Giacomini, A.: A variational approach to the isoperimetric inequality for the Robin eigenvalue problem. Arch. Ration. Mech. Anal. 198(3), 927-961 (2010)

6. Bucur, D., Giacomini, A.: Faber-Krahn inequalities for the Robin-Laplacian: a free discontinuity approach. Arch. Ration. Mech. Anal. 218(2), 757-824 (2015)

7. Bucur, D., Giacomini, A., Trebeschi, P.: Best constant in Poincaré inequalities with traces: a free discontinuity approach. Ann. Inst. H. Poincaré Anal. Non Linéaire 36(7), 1959-1986 (2019)

8. Lindqvist, P.: Addendum: on the equation $\operatorname{div}\left(|\nabla u|^{p-2} \nabla u\right)+\lambda|u|^{p-2} u=0$ " [Proc. Am. Math. Soc. 109(1), 157-164 (1990) MR1007505 (90h:35088)]. Proc. Am. Math. Soc. 116(2), 583-584 (1992)

9. Nečas, J.: Direct methods in the theory of elliptic equations. Springer Monographs in Mathematics. Springer, Heidelberg (2012). Translated from the 1967 French original by Gerard Tronel and Alois Kufner, Editorial coordination and preface by Šárka Nečasová and a contribution by Christian G. Simader

10. Talenti, G.: Elliptic equations and rearrangements. Ann. Scuola Norm. Sup. Pisa Cl. Sci. (4) 3(4), 697718 (1976)

11. Talenti, G.: Nonlinear elliptic equations, rearrangements of functions and Orlicz spaces. Ann. Mat. Pura Appl. 4(120), 160-184 (1979)

12. Talenti, G.: Inequalities in rearrangement invariant function spaces. In: Nonlinear Analysis, Function Spaces and Applications, vol. 5, pp. 177-230. Prometheus, Prague (1994)

13. Vázquez, J.L.: A strong maximum principle for some quasilinear elliptic equations. Appl. Math. Optim. 12(3), 191-202 (1984)

Publisher's Note Springer Nature remains neutral with regard to jurisdictional claims in published maps and institutional affiliations. 